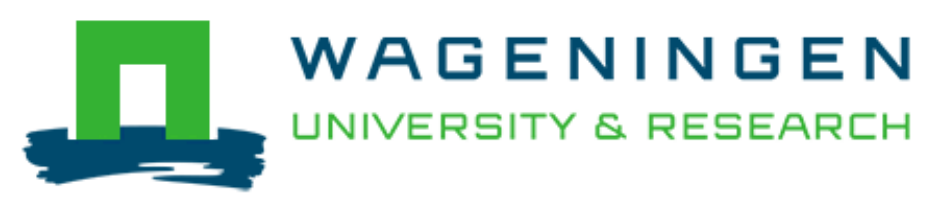

Two-fluid model for the simultaneous flow of colloids and fluids in porous media

\author{
Biesheuvel, P. M.
}

This is a "Post-Print" accepted manuscript, which has been published in "Journal of Colloid and Interface Science"

This version is distributed under a non-commercial no derivatives Creative Commons (C) $(1) \Theta($ (CC-BY-NC-ND) user license, which permits use, distribution, and reproduction in any medium, provided the original work is properly cited and not used for commercial purposes. Further, the restriction applies that if you remix, transform, or build upon the material, you may not distribute the modified material.

Please cite this publication as follows:

Biesheuvel, P. M. (2011). Two-fluid model for the simultaneous flow of colloids and fluids in porous media. Journal of Colloid and Interface Science, 355(2), 389-395. https://doi.org/10.1016/j.jcis.2010.12.006 


\title{
Two-fluid model for the simultaneous flow of colloids and fluids in porous media
}

P.M. Biesheuvel

Department of Environmental Technology, Wageningen University, Bomenweg 2, 6703 HD Wageningen, The Netherlands. Wetsus, Centre of Excellence for Sustainable Water Technology, Agora 1, 8934 CJ Leeuwarden, the Netherlands. E-mail: maarten.biesheuvel@wur.nl

\begin{abstract}
To describe the velocities of particles such as ions, protein molecules and colloids dispersed or dissolved in a fluid, it is important to also describe the forces acting on the fluid, including pressure gradients and friction of the fluid with the particles and with the porous media through which the fluid flows. To account for this problem, the use of a two-fluid model is described, familiar in the field of fluid mechanics, extended to include osmotic effects. We show how familiar relationships follow in various situations and give examples of combined fluid/particle transport in neutral and charged membranes driven by a combination of electrostatic, diffusional and pressure forces. The analysis shows how the same modeling framework can be generally used both for multidimensional electrokinetic flow through macroscopic channels and around macroscopic objects, as well as for mean-field modeling of transport through porous media such as gels and membranes.
\end{abstract}

One of the basic problems in electrokinetics, colloid science and membrane science is how to describe simultaneously the transport rates of particles (like ions, molecules, colloids) and the fluid in which the particles are dissolved or dispersed..$^{1-12}$ Examples abound such as the flow of aqueous solutions in microfluidic systems, ${ }^{13,14,15}$ desalination using electrical fields across porous electrodes, ${ }^{16-18}$ energy recovery from water salinity differences, ${ }^{19-22}$ and membrane processes for water treatment such as nanofiltraton and reverse osmosis. ${ }^{23-29}$ In such processes, several forces act on the fluid such as pressure and friction, while the particles are subject to forces such as concentration gradients and the electrical field. ${ }^{30}$ The fluid and the particle transport rates are not independent but couple through mutual friction, i.e., the transfer of momentum. How to describe this problem?

Here we suggest the following mathematical framework. First of all, the velocity $\mathbf{v}_{\mathbf{i}}$ of each of the particle types relative to the fluid can be described by a modified Nernst-Planck equation

$\mathbf{v}_{\mathrm{i}}-\mathbf{v}_{\mathrm{F}}=-D_{\mathrm{i}} \cdot\left(\nabla \ln c_{\mathrm{i}}+z_{\mathrm{i}} \nabla y+V_{\mathrm{i}} \nabla P^{\mathrm{t}}-m_{\mathrm{i}} \mathbf{g}+\nabla \mu_{\mathrm{i}}^{\mathrm{ex}}+\nabla \chi_{\mathrm{i}}\right)$

where $\mathbf{v}_{\mathrm{i}}$ is the velocity of particle type $\mathrm{i}$, and $\mathbf{v}_{\mathrm{F}}$ is the fluid velocity (typesetting in bold is used to denote vector quantities). $D_{\mathrm{i}}$ is the binary (fluid-particle) diffusion coefficient, $c_{\mathrm{i}}$ the concentration of the particles, $z_{i}$ unitary charge (e.g. +1 for a cation), $y$ the dimensionless electrical potential to be multiplied with $R T / F=k T / e$ to obtain the dimensional voltage, $V_{i}$ the volume per particle, $P^{t}$ the total pressure to be discussed below, $m_{\mathrm{i}}$ the mass per particle $\left(=\rho_{\mathrm{i}} \cdot V_{\mathrm{i}}\right.$, where $\rho_{\mathrm{i}}$ is the particle mass density), $\mathbf{g}$ an acceleration factor for gravity and centrifugation, $\mu^{\mathrm{ex}}$ an excess contribution to the chemical potential (equivalent to $\ln \gamma$, with $\gamma$ the activity coefficient), e.g. describing volumetric interactions between the particles, $\chi$ an interaction energy of the particle with the medium (solvation energy), and where $\nabla x$ denotes gradients in $x$, i.e., the change of $x$ with the spatial coordinate. Parameters $P^{t}, \mathbf{g}, \mu^{\mathrm{ex}}$ and $\chi$ are 
reduced quantities and need to be multiplied by the thermal energy, $k T$, to return to the conventional dimension of $P^{t}$ in $\mathrm{Pa}, \mathbf{g}$ in $\mathrm{m} / \mathrm{s}^{2}$, and $\mu^{\mathrm{ex}}$ and $\chi$ in $\mathrm{J}$.

Eq. (1) describes how the velocity difference of the particle with the fluid is proportional to a total force which has possible contributions from a concentration gradient (diffusional term), electrical fieldeffects, pressure gradients, gravity (centrifugation), an excess contribution for concentrated solutions, ${ }^{31,32}$ and a gradient in solvation energy. In Eq. (1) all frictional interactions go via the fluid, and thus we neglect direct particle-particle and particle-wall (or particle-matrix) frictions (ref. 1, p. 56).

Eq. (1) can be rewritten to give the particle flux, $\mathbf{J}_{\mathrm{i}}=c_{\mathrm{i}} \cdot \mathbf{v}_{\mathrm{F}}$, as a summation of a convective term, $c_{\mathrm{i}} \cdot \mathbf{v}_{\mathrm{F}}$, and a term due to the forces acting on the particle, ${ }^{1,28,33}$

$\mathbf{J}_{\mathrm{i}}=c_{\mathrm{i}} \mathbf{v}_{\mathrm{F}}-D_{\mathrm{i}} \cdot\left(\nabla c_{\mathrm{i}}+c_{\mathrm{i}} z_{\mathrm{i}} \nabla y+\phi_{\mathrm{i}} \nabla P^{\mathrm{t}}-c_{\mathrm{i}} m_{\mathrm{i}} \mathbf{g}+c_{\mathrm{i}} \nabla \mu_{\mathrm{i}}^{\mathrm{ex}}+c_{\mathrm{i}} \nabla \boldsymbol{\chi}_{\mathrm{i}}\right)$.

Next, an expression is required for the fluid velocity, $\mathbf{v}_{\mathrm{F}}$. To this end we propose the use of a modified two-fluid model, which is a modified Navier-Stokes equation for fully developed flow of a Newtonian fluid of constant viscosity and density where we include porosity effects and friction of the fluid with all dispersed particle types i, resulting in

$\left(\nabla P^{\mathrm{t}}-\rho_{\mathrm{F}} \mathbf{g}-\mu \nabla^{2} \mathbf{v}_{\mathrm{F}}\right) \cdot(1-\phi)=\sum_{\mathrm{i}} \frac{C_{\mathrm{i}}}{D_{\mathrm{i}}} \cdot\left(\mathbf{v}_{\mathrm{i}}-\mathbf{v}_{\mathrm{F}}\right)$

where the index i runs over all dispersed particles, $\rho_{F}$ is the fluid mass density, $\mu$ is the viscosity of the fluid and $\phi$ is the volume fraction of all particles combined $\left(\phi=\Sigma_{i} \phi_{i}, \phi_{i}=c_{i} \cdot V_{i}\right)$, i.e. $\phi$ is equal to one minus the porosity. Eq. (3) extends the two-fluid model from fluid mechanics ${ }^{34-37}$ by replacing the hydrostatic pressure by the total pressure (including osmotic effects) and by allowing for friction with multiple particle species. One of these species can have zero velocity and then represents the porous medium. Note that Eq. (3) only follows from the formal expression when the porosity, 1- $\phi$, is the same everywhere, which can be assumed for flow through a porous medium of constant density, with dispersed particles (ions) that can be approximated as being volumeless. Otherwise, the term $(1-\phi) \cdot \mu \nabla^{2} \mathbf{v}_{\mathrm{F}}$ in Eq. (3) must be replaced by the full expression for the viscous stress tensor, $\nabla(1-\phi) \cdot \tau$, see ref. 38, p. 349.

For flow through channels or around objects, Eq. (3) can be used and the fluid velocity set to zero at all solid walls. An important difference with literature on two-phase flow of non-colloidal particles ${ }^{34-37}$ is that we propose to use a total pressure $P^{t}$ in Eq. (3) instead of the hydrostatic pressure $P^{h}$. At equilibrium (when all $v_{i}^{\prime} S=0$, and $v_{F}=0$ ), and without external forces acting on the fluid such as gravity and centrifugation (i.e., for $\mathbf{g}=0$ ), Eq. (3) shows how the gradient in total pressure is zero, $\nabla P^{t}=0$, and thus in Eqs. (1) and (2) which at equilibrium describe the density distribution of the dispersed particles, the term $V_{i} \cdot \nabla P^{t}$ disappears. This simplification of Eq. (1) is in agreement e.g. with Poisson-Boltzmann double layer theory which describes the equilibrium ion distribution near charged interfaces. In the literature of two-phase flow (e.g. refs. 34,35), the term $\beta=c_{i} / D_{i}$ in Eq. (3) is sometimes empirically described by a modified Stokes-equation, e.g. for low Reynolds-numbers given by $\beta=18 \cdot \mu \cdot \phi_{i} / \sigma_{i}{ }^{2} /(1-\phi)^{2.65}$, where $\sigma_{i}$ is particle size. For multicomponent suspensions, more elaborate expressions are available. . $^{37,39,40}$

As Eqs. (1)-(3) show, to describe the fluid flow, we use a very different expression compared to that for the dispersed particles. This difference reflects how the fluid is fundamentally different from the 
particles, the fluid being continuous and having to fill at all times the space in between the particles, which are dispersed. Note for instance how electrostatic forces are included in Eqs. (1) and (2) and not in Eq. (3), as such forces only act directly on the ions and not on the fluid. However, interestingly, we can derive from Eq. (3) the classical expression used in electrokinetics for the relation between hydrostatic pressure gradients and electrostatic body forces, but only via coupling with the expressions for particle velocity, Eq. (1), as we will show below.

For transport through a porous medium (a 'matrix' phase) such as gels, membranes and porous electrodes, we can include the matrix, $m$, into the summation term on the right-hand side of Eq. (3), and set the velocity of the " $m$ "-particle to zero, $\left|\mathbf{v}_{\mathrm{m}}\right|=0$, thereby modeling the matrix as a collection of particles with positions fixed in space. For the matrix-fluid friction, we can then replace $c_{\mathrm{m}} / D_{\mathrm{m}}$ by a frictional coefficient $f_{\mathrm{m}}$. Interestingly, we then arrive at a generalization of the Brinkman equation, which describes fluid flow through porous media, next to fixed walls, or open channels. ${ }^{41,42,43}$

As we will show, the above equations can be recombined into familiar expressions for combined fluid and particle flow in various applications, but it is necessary to use the total pressure $P^{t}$ in Eqs. (1)-(3) and equate $P^{t}$ to the hydrostatic (or, hydraulic) pressure $P^{h}$ minus the osmotic pressure, $\Pi$, i.e., $P^{t}=P^{h}$ - . Note that this 'total pressure' is a classical concept, see Mauro, ${ }^{45}$ Ray ${ }^{46}$ and the 'solvent partial pressure' in Osterle. ${ }^{4,5}$ In many situations we can neglect $\Pi$ such as for non-colloidal suspensions of large particles and/or when non-osmotic forces are much larger, but when we make this assumption in general, then the physics of many classical problems cannot be explained, such as osmosis, the phenomenon where fluid moves against a hydraulic pressure gradient toward locations of higher osmotic pressure, $\Pi$. Also, to derive several well-known equations to be discussed below, it is necessary to include $\Pi$ in $P^{t}$. Thus, we argue that in Eqs. (1)-(3) $P^{t}$ must be a combination of a hydrostatic pressure $P^{h}$ and an osmotic contribution, $\Pi$.

As a first example, when we aim at describing electrokinetic flow through capillaries or around macroscopic objects, we can implement Eq. (1) in Eq. (3), which (when leaving out the solvation term) results in $1,4,7,30,47,48,49$

$\mu \nabla^{2} \mathbf{v}_{\mathrm{F}}=\nabla P^{\mathrm{h}}+\sum_{\mathrm{i}} z_{\mathrm{i}} c_{\mathrm{i}} \cdot \nabla y-\rho_{\mathrm{t}} \mathbf{g}$

which is familiar in physico-chemical hydrodynamics and describes how besides a pressure gradient and gravity, there is an electrostatic body force which acts on any fluid element that is locally not in charge-balance. Our analysis shows how this result can be derived from combination of Eqs. (1) and (3), without making the intuitively perhaps difficult assumption how an electrostatic body force directly acts on an uncharged fluid. In a full calculation, Eq. (4) can be combined with Eq. (1) for each of the particle types, as well as with Poisson's law, $\nabla^{2} y=-4 \pi \lambda_{B} \cdot \sum_{i} z_{i} c_{i}$ with $\lambda_{B}$ the Bjerrum length, and conservation balances for all particle types, $\partial c_{i} / \partial t=-\nabla\left(c_{i} \cdot \mathbf{v}_{i}\right)$. This set of equations then describes electrokinetics of incompressible fluids with dispersed particles, including particle volume effects, as long as direct particle-particle and particle-wall friction can be neglected (i.e., all frictional effects go via the fluid). Constitutive relations are required which describe how effective viscosity $\mu$ and diffusion coefficients $D_{\mathrm{i}}$ depend on particle volume fractions and velocities. ${ }^{40,50,51}$ 
From this point onward we focus on flow through porous media sufficiently far away from macroscopic edges of the porous medium with either open channels or a hard wall, such that we can leave out the fluid dissipation term $\mu \nabla^{2} \mathbf{v}_{\mathrm{F}}$ from Eq. (3), and describe fluid dissipation solely by the term $f_{\mathrm{m}} \cdot \mathbf{v}_{\mathrm{F}}$, which results in

$$
\left(\nabla P^{\mathrm{t}}-\rho_{\mathrm{F}} \mathbf{g}\right) \cdot(1-\phi)+f_{\mathrm{m}} \cdot \mathbf{v}_{\mathrm{F}}=\sum_{\mathrm{i}} \frac{C_{\mathrm{i}}}{D_{\mathrm{i}}} \cdot\left(\mathbf{v}_{\mathrm{i}}-\mathbf{v}_{\mathrm{F}}\right) .
$$

Eq. (5) describes how a pressure gradient and gravitational contributions acting on the fluid are balanced by friction of the fluid with a stagnant matrix and by friction with dispersed particles. Leaving out the term $f_{\mathrm{m}} \cdot \mathbf{v}_{\mathrm{F}}$ and dividing each side by $1-\phi$, Eq. (5) is given in ref. 44 to describe multicomponent fluidization and sedimentation, with the right-hand side of Eq. (5) then equal to the hydrodynamic drag force acting on the fluid.

For the simple case where we only have fluid (water) transport through a stationary matrix (thus $\Pi=0$ and all $c_{i}^{\prime}$ 's zero), without gravity-effects, Eq. (5) simplifies to the classical Darcy equation,

$\mathbf{v}_{\mathrm{F}}=-k_{\mathrm{m}} \nabla P^{\mathrm{h}}$

where the permeability $k_{\mathrm{m}}$ equals $(1-\phi) / f_{\mathrm{m}}$.

For more general situations, let us combine Eqs. (1) and (5) to obtain several familiar results and expressions. First of all, we include Eq. (1) in Eq. (5), and make use of the Gibbs-Duhem relationship ${ }^{9}$ $\nabla \Pi=\nabla \sum_{i} c_{i}+\sum_{i} c_{i} \nabla \mu_{i}^{e x}$

to obtain

$\nabla P^{\mathrm{h}}+f_{\mathrm{m}} \cdot \mathbf{v}_{\mathrm{F}}=-\sum_{\mathrm{i}} z_{\mathrm{i}} c_{\mathrm{i}} \cdot \nabla y+\rho_{\mathrm{t}} \mathbf{g}-\sum_{\mathrm{i}} c_{\mathrm{i}} \nabla \chi_{\mathrm{i}}$

where the average, suspension, density is $\rho_{\mathrm{t}}=\sum_{\mathrm{i}} \phi_{\mathrm{i}} \rho_{\mathrm{i}}+\rho_{\mathrm{F}}(1-\phi)$, where $\phi_{\mathrm{i}}=V_{\mathrm{i}} \cdot c_{\mathrm{i}}$.

At equilibrium, or in an unrestricted medium, without gravity and solvation effects, Eq. (8) simplifies to the well-known equality ${ }^{47}$

$\nabla P^{h}+\sum_{i} z_{i} c_{i} \cdot \nabla y=0$

often applied in the study of forces in electrostatic double layer theory.

For the flow of the dispersed particles, we first implement Eq. (8) in Eq. (1), and making use again of $\nabla P^{t}=\nabla P^{h}-\nabla \Pi$, we obtain the following rather unwieldy equation,

$\mathbf{v}_{\mathrm{i}}-\mathbf{v}_{\mathrm{F}}=-D_{\mathrm{i}} \cdot\left(\nabla \ln c_{\mathrm{i}}+z_{\mathrm{i}} \nabla y-V_{\mathrm{i}}\left(\nabla \Pi+\sum_{\mathrm{i}} z_{\mathrm{i}} c_{\mathrm{i}} \cdot \nabla y+\sum_{\mathrm{i}} c_{\mathrm{i}} \nabla \chi_{\mathrm{i}}+f_{\mathrm{m}} \cdot \mathbf{v}_{\mathrm{F}}\right)-V_{\mathrm{i}} \mathbf{g}\left(\rho_{\mathrm{i}}-\rho_{\mathrm{t}}\right)+\nabla \mu_{\mathrm{i}}^{\mathrm{ex}}+\nabla \chi_{\mathrm{i}}\right)$.

For mixtures of sedimenting non-colloidal (i.e., large) and uncharged particles without solvency and matrix effects (i.e., $\Pi=0, z=0, \chi=0, \mu^{\mathrm{ex}}=0, f_{\mathrm{m}}=0$ ), Eq. (10) then simplifies to ${ }^{31,39,44}$

$\mathbf{v}_{\mathrm{i}}-\mathbf{v}_{\mathrm{F}}=\frac{\sigma_{\mathrm{i}}^{2} \mathbf{g}^{*}}{18 \mu} h_{\mathrm{i}}\left(\rho_{\mathrm{i}}-\rho_{\mathrm{t}}\right)$

where $\sigma_{\mathrm{i}}$ is the particle size, $\mathbf{g}^{*}$ the dimensional acceleration factor $(=\mathbf{g} \cdot k T)$, and $h_{\mathrm{i}}$ a hindrance factor, describing the reduction of particle mobility in dense suspensions, which for mixtures where all particles have the same velocity and size can be described by the Richardson-Zaki equation, $h_{\mathrm{i}}=(1-\phi)^{n-2}$, 
with $n=4.65$ for laminar flow. To derive Eq. (11) we have made use of $D_{i}=\frac{k T}{3 \pi \sigma_{i} \mu} h_{1}$. Eqs. (10) and (11) correctly show how for sedimentation (centrifugation), the effective driving force on a particle is the mass density difference of the particle, $\rho_{\mathrm{i}}$, not with the pure fluid, $\rho_{\mathrm{F}}$, but with the mass density of the suspension, $\rho_{t}{ }^{31,39,44}$

Next we consider the equilibrium sedimentation profile of a multi-component molecular or colloidal mixture in a centrifugal or gravity field (e.g., the earth's atmosphere), i.e., all velocities are zero. Interestingly, whether the space (fluid) in between the particles (molecules) is empty, or an incompressible fluid, does not matter when equilibrium is considered (but is very important for transport modeling). As Eqs. (3) and (5) show, at equilibrium, when the right-hand side of Eqs. (3) and (5) is zero, the osmotic pressure gradient $\nabla \Pi$ exactly equals the hydrostatic pressure gradient $\nabla P^{h}$ minus the fluid pressure gradient $\rho_{\mathrm{F}} \cdot \mathbf{g}^{52}$

$\nabla P^{\mathrm{h}}=\nabla \Pi+\rho_{\mathrm{F}} \mathbf{g}$.

Because the hydrostatic pressure gradient must be equal to

$\nabla P^{\mathrm{h}}=\rho_{\llcorner} \mathbf{g}(1-\phi)+\sum_{\mathrm{i}} \rho_{\mathrm{i}} \phi_{\mathrm{i}} \mathbf{g}=\rho_{\mathrm{t}} \mathbf{g}$

we can, in a multicomponent colloidal mixture, directly obtain the pressure gradient $\nabla P^{\mathrm{h}}$ from the measured density profiles $\phi_{1}(h) \ldots \phi_{\mathrm{n}}(h)$ as function of height $h$, and thus measure the osmotic pressure gradient, $\nabla \Pi$, allowing to reconstruct the equation-of-state $\Pi\left(\phi_{1} \ldots \phi_{n}\right)$. That the pressure gradient $\nabla P^{\mathrm{h}}$ must necessarily be given by Eq. 13 is in line with the above theory, as shown by inserting $\mathbf{v}_{\mathrm{i}}-\mathbf{v}_{\mathrm{F}}=0, z=0, \chi=0$ and $f_{\mathrm{m}}=0$ in Eq. (10), multiplying by $c_{\mathrm{i}}$ and adding up for all particle species, which leads to $(1-\phi) \nabla \Pi=\sum_{i} \phi_{i}\left(\rho_{i}-\rho_{t}\right)$ g which can be combined with Eq. 12 to result in Eq. 13 .

It is possible to theoretically predict the equilibrium density profiles for colloidal systems. E.g., for a one-component mixture of hard-sphere particles, we can use the Carnahan-Starling equation of state to describe the excess contribution to the potential, given by $\mu^{\mathrm{ex}, \mathrm{CS}}=(3-\phi) \cdot(1-\phi)^{-3}-3$ and $\Pi^{\mathrm{CS}} \cdot V_{\mathrm{i}}=\phi\left(1+\phi+\phi^{2}-\phi^{3}\right) \cdot(1-\phi)^{-3} \quad$ (refs. 31,32,52) and obtain from Eq. (10) the equality $\nabla \Pi=\nabla c_{\mathrm{i}}+c_{\mathrm{i}} \nabla \mu_{\mathrm{i}}^{\mathrm{ex}}=\phi \mathbf{g}\left(\rho_{\mathrm{i}}-\rho_{\mathrm{F}}\right)$, resulting in the implicit expression for concentration versus height given by

$\ln \frac{c}{c_{0}}+\frac{3-\phi}{(1-\phi)^{3}}-\frac{3-\phi_{0}}{\left(1-\phi_{0}\right)^{3}}=m_{\mathrm{b}, \mathrm{i}} \mathbf{g} h$

with $c_{0}$ and $\phi_{0}$ conditions at $h=0$ (note, $\phi=c \cdot V_{\mathrm{i}}$ ), and $m_{\mathrm{b}, \mathrm{i}}$ the particle mass relative to the fluid, $m_{\mathrm{b}, \mathrm{i}}=V_{\mathrm{i}} \cdot\left(\rho_{\mathrm{i}}-\rho_{\mathrm{F}}\right)$; note here that $\mathbf{g}<0$ when the coordinate $h$ points upward in earth's gravity field. For low densities (when $\mu^{\mathrm{ex}}=0$ ), Eq. (14) result in the classical barometric profile for density $c=c_{0} \cdot \exp \left(m_{\mathrm{b}, \mathrm{g}} \mathbf{g} h\right)$, and (osmotic) pressure $\Pi_{0}-\Pi(h)=c_{0} \cdot\left(1-\exp \left(m_{\mathrm{b}, \mathbf{i}} \mathbf{g} h\right)\right)$.

Next we will apply Eqs. (1)-(10) to two classical membrane problems where osmotic, electrostatic, hydrostatic and solvency-effects all play a role. We neglect volume effects of the dispersed particles 
(i.e., $V_{i}=0, \mu_{i}^{e x}=0$ ) and only discuss planar membranes and steady-state transport problems. We also neglect gravity.

\section{Example l. Ultrafiltration: fluid and particle transport through a neutral membrane.}

In this example, we consider a planar homogeneous membrane of thickness $\delta$ placed in between two bulk phases with unequal hydraulic pressure $P_{\mathrm{j}}^{\mathrm{h}, \infty}$ and unequal concentration of a neutral particle (solute) $c_{j}^{\infty}$ (where $\mathrm{j}$ is "L" for the left compartment and " $\mathrm{R}$ " for the right compartment). The superscript " $\infty$ " stands for the bulk phase outside the membrane, while " $m$ " describes concentrations and pressures inside the membrane. We neglect the stagnant diffusion layers (Nernst layers, mass transfer films) on either side of the membrane. In steady state the flux of the solute, $J_{i}=c_{i} \cdot v_{i}$, is constant across the membrane. We assume also that the fluid velocity $v_{\mathrm{F}}$ is constant across the membrane, which requires that the solute particles are without volume (and the membrane is everywhere equally open). Otherwise the gradients that develop in the solute concentration and the membrane density lead to a gradient in open pore space and thus -for the fluid flow rate to remain constant- this would lead to a gradient in fluid velocity $v_{\mathrm{F}}$. These effects are presently not included.

Eq. (1) can be applied to describe solute flow within the bulk of the membrane, as well as for the membrane edges where we assume that gradients are steep over a very thin layer. There we can assume (quasi-)equilibrium which implies that the sum of forces in Eq. (1) adds up to zero, or equivalently, that the total potential for the solute, $\mu$, is constant across the membrane/solution-boundary. For a neutral membrane and neutral solute, this results in $c_{j}^{m} / c_{j}^{\infty}=\exp (-\Delta \chi)=S$, where $\Delta \chi=\chi^{m}-\chi^{\infty}$, and $S$ is the socalled sieving coefficient. Implementing this relation in Eq. (8) leads to

$\nabla P_{j}^{h}+c \nabla \chi(x)=\nabla P_{j}^{h}(x)-c_{j}^{\infty} \nabla \exp (-\chi(x))=0$,

which can be integrated across the interface, resulting in

$\Delta P_{j}^{h, i n t}-c_{j}^{\infty}[\exp (-\Delta \chi)-1]=\Delta P_{j}^{\text {h,int }}+c_{j}^{\infty}[1-S]=0$

where $\Delta P_{\mathrm{j}}^{\mathrm{h}, \mathrm{int}}=P_{\mathrm{j}}^{\mathrm{h}, \mathrm{m}}-P_{\mathrm{j}}^{\mathrm{h}, \infty}$. Thus, with $\Delta \chi$-the solvation energy increase for a particle to enter the membrane- positive, we have $0<S<1$, i.e., the concentration of particles just within the membrane is lower than in the adjacent solution, which results in a decrease both in the osmotic and hydraulic pressure upon entering the membrane (with the total pressure $P^{t}$ remaining constant across the interface). ${ }^{1,7,8,46}$

Within the membrane, $\chi$ is constant and we can integrate Eq. (1) across the membrane to obtain ${ }^{1,8,53}$

$J_{i}=v_{F} \frac{c_{L}^{m} \exp \left(v_{F} x D_{i}^{-1}\right)-c^{m}(x)}{\exp \left(v_{F} x D_{i}^{-1}\right)-1}$.

Particle flux $J_{i}$ follows explicitly when $x=\delta$ is inserted. Eq. (17) can be rewritten to an explicit expression for the particle concentration profile in the membrane ${ }^{53}$

$c^{m}(x)=S \frac{\left(c_{\mathrm{L}}^{\infty} \exp \left(v_{\mathrm{F}} \delta D_{i}^{-1}\right)-c_{\mathrm{R}}^{\infty}\right)-\left(c_{\mathrm{L}}^{\infty}-c_{\mathrm{R}}^{\infty}\right) \exp \left(v_{\mathrm{F}} x D_{i}^{-1}\right)}{\exp \left(v_{\mathrm{F}} \delta D_{\mathrm{i}}^{-1}\right)-1}$. 
This solution of Eq. (1), i.e., the concentration profile due to the superposition of a diffuse Fickian flux on a convective contribution, was first given by Hertz. ${ }^{54}$ Eq. (18) predicts that when the fluid velocity goes to zero $\left(v_{\mathrm{F}} \rightarrow 0\right)$, then, making use of $\exp (x) \rightarrow 1+x$, the particle flux becomes $J_{\mathrm{i}}=S \cdot D_{\mathrm{i}} / \delta \cdot\left(C_{\mathrm{L}}{ }^{\infty}-C_{\mathrm{R}}{ }^{\infty}\right)$, which is the classical expression for simple diffusion through a stagnant medium, with $S$ the 'solubility', $D_{\mathrm{i}}$ mobility, $\delta$ thickness, and the driving force being the linear concentration difference, $\Delta c^{\infty}$. In this case the concentration profile also decays linearly (see the curve labelled $v_{\mathrm{F}} \cdot D_{\mathrm{i}} / \delta=0$ in Fig. $1 \mathrm{a}$ ). Eqs. (17) and (18) show how flow of fluid modifies this simple behavior, even for the simple problem of steady-state diffusion through a stagnant matrix phase.

In Fig. 1 we show calculation results based on Eqs. (17) and (18) as function of the dimensionless group $v_{\mathrm{F}} \cdot D_{\mathrm{i}} / \delta$. As Fig. 1a shows, only for a zero fluid velocity is the classical linear decay of concentration observed, while positive fluid velocities 'push' the profile 'outward', and vice-versa for negative fluid velocities, similar to Fig. 10 in ref. 1, and Fig. 10 in ref. 53.

In Fig. $1 \mathrm{~b}$ (related to Fig. 3 in ref. 55), the particle flux $J_{i}$ is plotted versus fluid velocity $v_{F}$ and we observe how for a zero particle concentration in the right bulk the particle flux is always positive (directed to the right) irrespective of fluid velocity, and thus is oriented opposite to the fluid velocity for $v_{F}<0$. The range of fluid velocities $v_{F}$ where particles and fluid move in opposite direction (i.e., where $J_{i}$ is positive and $v_{\mathrm{F}}$ negative) shrinks with increasing $c_{\mathrm{R}}{ }^{\infty}$, but disappears only when $c_{\mathrm{R}}{ }^{\infty}=C_{\mathrm{L}}$.

Next we calculate $v_{\mathrm{F}}$ which according to Eq. (8) is simply

$$
v_{\mathrm{F}}=\left(f_{\mathrm{m}} \delta\right)^{-1} \cdot \Delta P^{\mathrm{h}, \mathrm{m}}
$$

where $\Delta P^{\mathrm{h}, \mathrm{m}}=P_{\mathrm{L}}^{\mathrm{h}, \mathrm{m}}-P_{\mathrm{R}}^{\mathrm{h}, \mathrm{m}}$; i.e., in the membrane fluid flow is simply due to a hydraulic pressure gradient. But what about an osmotic effect influencing fluid flow? Interestingly, the osmotic effect follows from the solution/membrane boundaries where (when $S<1$ ) the hydraulic pressure in the membrane is decreased relative to outside, the more so at the side with the highest outside concentration, i.e., where $c_{j}^{\infty}$ is highest. Thus, (when $S<1$ ) different outside solute concentrations lead to a contribution to $\Delta P^{m}$ that works to push fluid in the direction of the high-concentration side. Implementing the above derived equality, $\Delta P_{\mathrm{j}}^{\mathrm{h}, \text { int }}=-c_{\mathrm{j}}^{\infty}(1-S)$, across each interface, we arrive at

$$
v_{\mathrm{F}}=\left(f_{\mathrm{m}} \delta\right)^{-1} \cdot\left\{\Delta P^{\mathrm{h}, \infty}-(1-S) \Delta \Pi^{\infty}\right\}
$$

where $\Delta P^{\mathrm{h}, \infty}=P_{\mathrm{L}}^{\mathrm{h}, \infty}-P_{\mathrm{R}}^{\mathrm{h}, \infty}$ and $\Delta \Pi^{\infty}=\Pi_{\mathrm{L}}^{\infty}-\Pi_{\mathrm{R}}^{\infty}=c_{\mathrm{L}}^{\infty}-c_{\mathrm{R}}^{\infty}$ and $\delta$ is membrane thickness. To have osmotically driven flow of fluid (i.e., fluid flow to the concentrated solution phase, in the absence of a hydraulic pressure difference), Eq. (20) shows that $S<1$ is required, i.e., the concentration of dispersed particles in the membrane must be below that in the bulk phases outside the membrane.

Eq. (20) can also be found in literature discussing phenomenological approaches based on linear flux-force relationships ${ }^{55-61}$ for membrane transport, with the group 1-S replaced by the reflection coefficient $\sigma$. The above analysis shows how the same relation also follows from Eqs. (1) and (5) and provides us with a clear physical definition of $\sigma$, namely being dependent only on an energy penalty for a particle to enter the membrane, independent on further process parameters, such as velocities, pressures or concentrations. Instead, in the literature on phenomenological approaches to membrane transport, the description of $\sigma$ is generally much more ambiguous, see e.g. ref. 57 , pp. 59-66. More 
generally, for problems of combined diffusion and convection, the phenomenological approach has been criticized for its significant deviation from models that describe transport microscopically, even for low driving forces, and even for the simple problem of Fickean diffusion combined with convective transport, i.e. the phenomenological approach does not agree with the Hertz equation, Eq. (17)., ${ }^{3,62-67}$
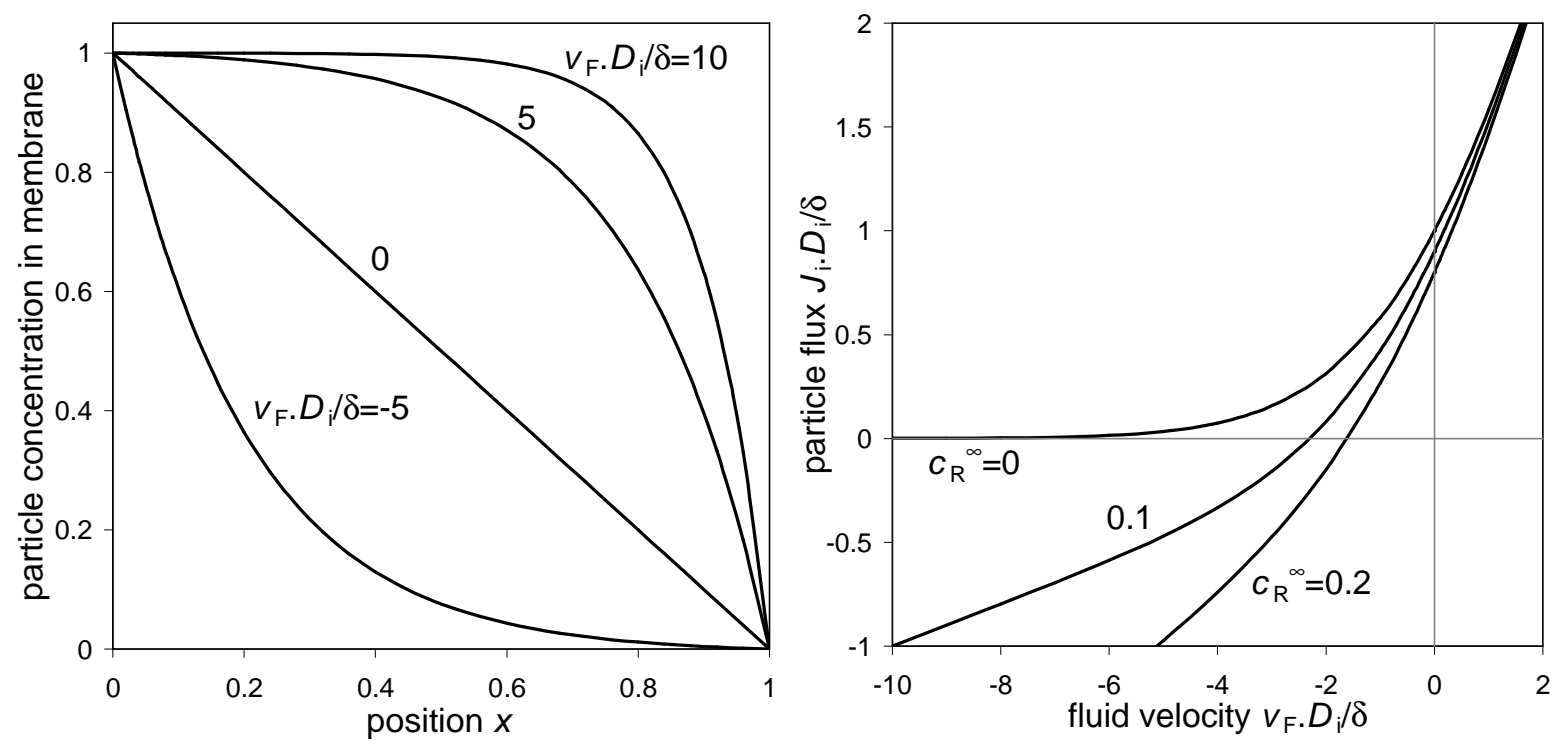

Fig. 1. Transport of a single solute through a neutral membrane. Concentration profiles and fluxes in a membrane separating two bulk phases with different particle concentration as function of the parameter $v_{\mathrm{F}} \cdot D_{\mathrm{i}} / \delta\left(S=1, c_{\mathrm{L}}{ }^{\infty}=1\right)$. (a). Particle concentration in membrane $\left(c_{\mathrm{R}}{ }^{\infty}=0\right)$. (b). Particle flux vs. fluid velocity.

\section{Example II. Osmotic flow across ion-exchange membrane.}

In the second example we consider steady-state electro-osmotic flow through an an ion-exchange membrane. We consider a 1:1 salt solution on both sides of the membrane and allow access of both anions and cations into the membrane. We take equal diffusivities of both ions and assume absence of effects of solvation, gravitation or ion-volume (or other non-idealities), thus $\chi=0, \mathbf{g}=0$ and $\mu^{\mathrm{ex}}=0$. The membrane has a concentration $X$ of fixed charges (the ion-exchange capacity) with charge-sign $\omega$ (e.g., $\omega=-1$ for a cation exchange membrane) ${ }^{68}$ Assuming local electroneutrality, at each position in the membrane we have $c^{+}-c^{-}+\omega X=0$ thus $\nabla c^{+}=\nabla c^{-}$, while zero net current implies $J_{=} J_{+}=J_{-}$. Thus, we do not consider the case of electrodialysis where a net current flows through the membrane, but instead only consider situations where cation- and anion-fluxes are equal, such as for pressure-driven or osmotically-driven flow. Driving forces considered are a difference in hydraulic pressure, osmotic pressure differences (i.e., differences in salt concentration) and resulting electrostatic effects.

For these assumptions, Eq. (8) results in $\mathrm{in}^{1,49,69}$

$\nabla P^{\mathrm{h}, \mathrm{m}}+f_{\mathrm{m}} \cdot v_{\mathrm{F}}=\omega X \cdot \nabla y^{\mathrm{m}}$

while Eq. (2) simplifies for both ions to ${ }^{2}$

$J_{\mathrm{i}}=c_{\mathrm{i}} v_{\mathrm{F}}-D_{\mathrm{i}} \cdot\left(\nabla c_{\mathrm{i}}+c_{\mathrm{i}} z_{\mathrm{i}} \nabla y\right)$.



$\nabla c^{+}=\nabla c^{-}=\frac{2 v_{F}}{D} \frac{c^{+} c^{-}}{c^{+}+c^{-}}-\frac{J}{D}$, 
$\nabla y=-\frac{\omega X}{c^{+}+c^{-}} \frac{v_{\mathrm{F}}}{D}$.

Applied to the solution-membrane boundaries, Eq. (8) results in

$\nabla P^{h}(x)+\sum_{\mathrm{i}} z_{\mathrm{i}} c_{\mathrm{i}}(x) \cdot \nabla y(x)=0$.

Here we can implement the Boltzmann equilibrium, $c_{\mathrm{i}}=c_{\mathrm{i}, \infty} \cdot \exp \left(-z_{\mathrm{i}} \cdot y_{\mathrm{i}}\right)$ resulting in

$\nabla P^{h}(x)-2 c_{j}^{\infty} \cdot \nabla \cosh (y(x))=0$

which we can integrate to

$\Delta P_{j}^{\mathrm{h}, \mathrm{int}}=2 c_{\mathrm{j}}^{\infty} \cdot\left(\cosh \left(\Delta y_{\mathrm{j}}^{\mathrm{int}}\right)-1\right)=\bar{c}_{\mathrm{j}}^{\mathrm{m}}-2 c_{\mathrm{j}}^{\infty}=\Delta \Pi_{\mathrm{j}}^{\mathrm{int}}$

where $\Delta y_{j}^{\text {int }}=y_{j}^{m}-y_{j}^{\infty}$ and $\bar{c}_{j}^{m}$ is the total (position-dependent) ion concentration in the membrane $\left(\bar{c}_{j}^{m}=c^{m,+}+c^{m,-}\right)$, here evaluated at the two solution/membrane interfaces. Eq. (27) shows how the osmotic pressure difference across the interface equals the hydraulic pressure difference, and thus the total pressure $P^{t}=P^{h}-\Pi$ remains invariant across the interface. ${ }^{2,7}$ The higher ion concentration in the ion-exchange membrane (relative to outside) leads to an increase in osmotic pressure across the interface equal to the increase in hydraulic pressure. It is at the side of the membrane where the ionconcentration outside the membrane is lowest, that the increase in hydraulic pressure is highest. Thus, osmotic effects across each membrane-solution interface lead to the development of a hydraulic pressure gradient within the membrane, pushing fluid to the high-concentration side (i.e., osmosis is positive). ${ }^{1,7}$

With

$\omega X=2 c_{j}^{\infty} \sinh \left(\Delta y_{j}^{\text {int }}\right)$

at each solution/membrane-interface, the pressure increase across the interface can also be calculated as ${ }^{1}$

$\Delta P_{j}^{\text {hint }}=\Delta \Pi_{j}^{\text {int }}=\sqrt{X^{2}+\left(2 c_{j}^{\infty}\right)^{2}}-2 c_{j}^{\infty}$,

i.e., is a direct function of outside ionic strength and membrane charge.

Assuming $v_{\mathrm{F}}$ to be constant, as in Example l, we can integrate Eq. (21) across the interior coordinate of the membrane to obtain ${ }^{1}$

$v_{\mathrm{F}}=\left(f_{\mathrm{m}} \delta\right)^{-1}\left(\Delta P^{\mathrm{h}, \mathrm{m}}-\omega X \cdot \Delta y^{\mathrm{m}}\right)$

where $\Delta y^{m}=y^{m} L-y^{m}$. Eq. (30) is an extension of Eq. (19) and shows that within the membrane hydrostatic and electrostatic effects drive the fluid, not osmotic effects.

In a practical calculation, we can subtract the pressure drops across both membrane interfaces from the total hydraulic pressure difference between the two bulk solutions, and obtain the internal membrane hydraulic pressure drop, $\Delta P^{h, m}$ which must be used in Eq. (30). Across the membrane, Eq. (23) can be integrated (after implementing $c^{+}-c^{-}+\omega X=0$ ) to obtain an analytical, but implicit, solution for concentration $c$ vs. position $x$. However, inserting that result in Eq. (24) and integrating for potential $y$ then proves impossible. 
Highly interestingly, Schlögl ${ }^{1,2}$ has obtained an exact, semi-analytical, solution for this problem, i.e., a solution for Eqs. (23) and (24), requiring two additional equations, as well as the two dummy variables $r$ and $s$. Even more admirable is that his solution also includes the situation of unequal coion and counterion diffusion coefficients, and also includes a possible current through the membrane (nonequal fluxes of cations and anions), i.e., the case of electrodialysis. Therefore, these equations by Schlögl ${ }^{1,2}$ are a very signficant extension of the classical semi-analytical models for ion-exchange membranes where the solvent (fluid) velocity is set to zero and pressure effects are not considered. ${ }^{56,57,70,71,72}$ Those theories are valid for normal osmosis and electrodialysis with the assumption of negligible fluid flow, and are therefore extended in Schlögl's formalism to also consider reverse (pressure driven) osmosis. Schlögl also describes how at both membrane edges we have jumps in concentration and hydrostatic pressure. Schlögl's work (which is in German) is not often mentioned in the english literature - some exceptions are the books by Helfferich ${ }^{73}$ and Lakshminarayanaiah. ${ }^{74}$

Following refs. 1 and 2, we can describe the steady-state transport of a mixture of cations and anions across a charged planar membrane by

$$
\begin{aligned}
& \Delta y^{\mathrm{m}}=\frac{r(U-\omega s)}{r-s} \ln \frac{\bar{c}_{\mathrm{R}}^{\mathrm{m}}-r X}{\overline{\mathrm{C}}_{\mathrm{L}}^{\mathrm{m}}-r X}-\frac{s(U-\omega r)}{r-s} \ln \frac{\bar{c}_{\mathrm{R}}^{\mathrm{m}}-s X}{{\overline{\mathrm{C}_{\mathrm{L}}^{\mathrm{m}}}-s X}}, \\
& \frac{v_{\mathrm{F}} \delta}{2}\left(\frac{1}{D^{+}}+\frac{1}{D^{-}}\right)=\frac{r}{r-s} \ln \frac{\bar{c}_{\mathrm{R}}^{\mathrm{m}}-r X}{\bar{c}_{\mathrm{L}}^{\mathrm{m}}-r X}-\frac{s}{r-s} \ln \frac{\bar{c}_{\mathrm{R}}^{\mathrm{m}}-s X}{\bar{c}_{\mathrm{L}}^{\mathrm{m}}-s X}, \\
& J^{+}=-\frac{v_{\mathrm{F}} \omega X}{4}\left(1+\frac{D^{+}}{D^{-}}\right)(\omega-r)(\omega-s), \\
& J^{-}=\frac{v_{\mathrm{F}} \omega X}{4}\left(1+\frac{D^{-}}{D^{+}}\right)(\omega+r)(\omega+s), \text { and } \\
& I=F \cdot\left(J^{+}-J^{-}\right)=-F v_{\mathrm{F}} \omega X \frac{\left(D^{+}+D^{-}\right)^{2}}{4 D^{+} D^{-}}\{1-\omega U(r+s)+r s\},
\end{aligned}
$$

where $r$ and $s$ are related according to

$1+U(r+s)+r s=0$

and $U$ is given by

$U=\frac{D^{+}-D^{-}}{D^{+}+D^{-}}$.

This set of 9 explicit equations (Eqs. 30-37) gives a full description for ion-exchange membrane transport with the 9 unknowns $r, s, U, J^{+}, J, l, v_{\mathrm{F}}, \Delta P^{\mathrm{h}, \mathrm{m}}$ and $\Delta y^{\mathrm{m}}$. The two parameters $\Delta P^{\mathrm{h}, \mathrm{m}}$ and $\Delta y^{\mathrm{m}}$ are related to conditions outside the membrane using Eqs. 28 and 29, while the salt concentration, $\bar{c}_{\mathrm{R}}^{\mathrm{m}}$, just within the membrane, at the edges, relate to outside concentrations according to $\bar{C}_{\mathrm{R}}^{\mathrm{m}}=\sqrt{X^{2}+\left(2 c_{\mathrm{j}}^{\infty}\right)^{2}}$. In any membrane process, two of the six operational parameters $J^{+}, J, I, v_{\mathrm{F}}, \Delta P^{\mathrm{h}, \mathrm{m}}$ (or $\Delta P^{h, \infty}$ ) and $\Delta y^{m}$ (or $\Delta y^{\infty}$ ) are prescribed, e.g., zero current, $l=0$, and a certain value for $\Delta P^{h, \infty}$ are prescribed in a nanofiltration- or reverse osmosis-process. Eqs. 30-37 can thus be considered to be the semi-analytical solution to the problem of steady-state flow through an ion-exchange membrane that allows passage of both ions, as well as allowing for fluid flow, including osmotic, electrostatic and 
pressure effects. This model is very broadly applicable and does not seem to be "extremely difficult to apply in practice" (ref. 57, p. 53).

In the case of zero current $(l=0)$, and assuming also $U=0$ (equal ion diffusivities), thus $r=-1 / s$, Eqs. 31-37 simplify to

$v_{\mathrm{F}} \delta\left(\frac{r}{D}-\frac{f_{\mathrm{m}}}{X}\right)=r \ln \frac{{\overline{C_{\mathrm{R}}^{\mathrm{m}}}}^{\mathrm{m}}-r X}{\overline{\mathrm{C}}_{\mathrm{L}}^{\mathrm{m}}-r X}-\frac{\Delta P^{\mathrm{h}, \mathrm{m}}}{X}$,

$v_{\mathrm{F}} \delta\left(\frac{s}{D}-\frac{f_{\mathrm{m}}}{X}\right)=s \ln \frac{{\overline{C_{\mathrm{R}}^{\mathrm{m}}}}^{\mathrm{m}}-s X}{{\overline{C_{\mathrm{L}}^{\mathrm{m}}}}^{\mathrm{m}}-s X}-\frac{\Delta P^{\mathrm{h}, \mathrm{m}}}{X}$,

$J=J^{+}=J^{-}=\frac{1}{2} v_{F} X(r+s)$.

For the condition of $l=0$ and $U=0$, we compared Schlögl's solution, Eqs. 30, 38-40, to the full numerical calculation based on Eqs. 23-30 and they gave exactly identical results.

Results from this model (for $l=0$ and $U=0$ ) are reported in Fig. 2 as full solid lines to be discussed below. Let us first discuss a simplified solution valid when the membrane charge density is much larger than the outside salt concentration (on both sides), $X>>c_{j}^{\infty}$. In this case we have $c^{\mathrm{m}, \text { counterion }} \sim X>>c^{\mathrm{m}, \mathrm{co}-\text { ion }}$, which allows us to solve for the co-ion concentration in the membrane, $c^{\text {m,co-ion }}$, as

$c^{m, \text { co-ion }}(x)=\left(2 v_{F}\right)^{-1}\left\{J+\left(2 v_{F} c_{L}^{m, c o-i o n}-J\right) \exp \left(2 v_{F} x / D\right)\right\}$,

and thus salt flux $J$ is given by ${ }^{7}$

$J=2 v_{\mathrm{F}} \frac{c_{\mathrm{R}}^{\mathrm{m}, \text { co-ion }}-c_{\mathrm{L}}^{\mathrm{m}, \mathrm{co-ion}} \exp \left(2 v_{\mathrm{F}} \delta / D\right)}{1-\exp \left(2 v_{\mathrm{F}} \delta / D\right)}=\frac{2 v_{\mathrm{F}}}{X} c_{\mathrm{L}}^{\infty} c_{\mathrm{R}}^{\infty} \frac{\sinh \left(v_{\mathrm{F}} \delta / D+\ln \left(c_{\mathrm{L}}^{\infty} / c_{\mathrm{R}}^{\infty}\right)\right)}{\sinh \left(v_{\mathrm{F}} \delta / D\right)}$

where at the solution/membrane-interfaces use is made of $c_{j}^{c 0-i o n,-}=\left(c_{j}^{\infty}\right)^{2} / X$, valid in this limit of a strongly charged ion-exchange membrane. To calculate $v_{F}$, we make use of Eq. (30) which in this limit of a high membrane charge results in

$v_{\mathrm{F}}=\left(f_{\mathrm{m}} \delta\right)^{-1}\left(\Delta P^{\mathrm{h}, \infty}-\Delta \Pi^{\infty}-\omega X \cdot \Delta y^{\mathrm{m}}\right)$

where $\Delta \Pi^{\infty}=2\left(c_{\mathrm{L}}^{\infty}-c_{\mathrm{R}}^{\infty}\right)$. Implementing Eq. (24), which for a highly charged ion exchange membrane is given by $D \cdot \nabla y=-\omega v_{F}$, we arrive at ${ }^{7}$

$v_{\mathrm{F}} \delta=\frac{\Delta P^{\mathrm{h}, \infty}-\Delta \Pi^{\infty}}{f_{\mathrm{m}}+X / D}$,

describing how the fluid flow is driven by a hydrostatic pressure difference minus an osmotic contribution. These expressions are similar to those by Sonin (ref. 7) who also describes general $z: z$ salts and unequal ion diffusivities. Eq. (44) shows that in this limit where the co-ion is blocked from the membrane, the full osmotic driving force is effective (as if the reflection coefficient $\sigma=1$ ), which is not the case in the general case of finite values of the ratio of membrane charge $X$ to outside salt concentration. Furthermore, Eq. (44) shows how in this limit of high ratios of $X$ over $c^{\infty}$, the fluid flow is a direct function of two resistances which are placed in series, namely the fluid-membrane friction (described by the resistance factor $f_{\mathrm{m}}$ ), and the fluid-ion friction. This fluid-ion friction is due to friction of the fluid 
with the almost stagnant counterions in the membrane, which have a negligible velocity because their concentration is much higher than that of the co-ion, namely by a factor $\left(X / c^{\infty}\right)^{2}$. This ion-fluid friction is proportional to the concentration of counterions (which is $\sim X$ ) and inversely proportional to the diffusion coefficient of the co-ion through the fluid-filled pores.

In the limit of $X / c_{\infty}$ large and equal ion diffusivities, Eqs. (42) and (44) describe the full membrane problem for combined fluid and salt transport through an ion-exchange membrane, which can be combined with analytical models for the concentration-polarization layers on both sides of the membrane and with mass balances in the bulk compartments. When we additionally assume high values for the fluid flow rate $v_{F}$, we can replace in Eq. (42) the sinh-terms with exp-terms, resulting in

$J=\frac{2 v_{F}}{X}\left(c_{L}^{\infty}\right)^{2}$

suggesting that under these conditions the salt flux becomes a linear function of fluid flow rate (which is in contrast to results of experiments reported in ref. 75 for $\mathrm{NaCl}$ transport in $\mathrm{RO}$ membranes).

Note that in a typical "dead-end" batchscale experiment there is no fixed concentration on the downstream-side ("R") of the membrane, but instead $c_{R}^{\infty}$ is self-consistently determined by the salt and fluid fluxes through the membrane, i.e., $C_{\mathrm{R}}^{\infty}=J / v_{\mathrm{F}} \cdot{ }^{7,8,76} \mathrm{In}$ such an experiment and for sufficiently high fluid flow rates $v_{F}$, Eq. (44) then predicts for the effluent salt concentration the value

$c_{\mathrm{R}}^{\infty}=J / v_{\mathrm{F}}=2\left(c_{\mathrm{L}}^{\infty}\right)^{2} / X$

independent of ion velocity and fluid flow rate.

Eq. (46) shows that to obtain a low effluent concentration, $c_{\mathrm{R}}^{\infty}$, high charge densities $X$ are favorable, but at the same time, as Eq. (44) shows, it must be realized that with increasing $X$ the resistance to fluid flow also increases. Eq. (46) can be rewritten to give the membrane retention $R$ as $R=1-\frac{C_{\mathrm{R}}^{\infty}}{C_{\mathrm{L}}^{\infty}}=1-2 c_{\mathrm{L}}^{\infty} / X$, i.e., the retention decreases with increasing upstream ionic strength. This term, $2 c_{\mathrm{L}}^{\infty} / X$, can be considered a limiting sieving coefficient. ${ }^{8}$ That retention is predicted to decrease with increasing upstream salt concentration is in agreement with experimental data reported in ref. 77 (fig. 7) and ref. 78 (fig. 6) but not with those reported in ref. 79 (fig. 2).

In Fig. 2 we show results for the downstream salt concentration $c_{R}^{\infty}$ as function of the applied pressure difference and membrane charge, for a reverse osmosis membrane placed in a dead-end batch cell. In such an experiment, $c_{R}^{\infty}$ will always be below the upstream concentration $c_{L}^{\infty}$. In Fig. 2 we compare full model calculations from Eqs. (30)-(40), with results of the analytical model given by Eqs. (42) and (44) (valid for high membrane charge $X$ ) and with the limiting expression, Eq. (46), valid in the limit of both high $X$ and $\Delta P^{\mathrm{h}, \infty}$. In Fig. 2a we see how with increasing pressure the effluent concentration first decreases and ultimately levels off when we arrive in the limiting regime where Eq. (46) well describes the downstream concentration. In Fig. $2 \mathrm{~b}$ we observe how results of the analytical expressions, Eqs. (42)-(46), are close to results of the full "Schlögl" model at sufficiently high $X$, but predict $c_{\mathrm{R}}^{\infty}$ to increase to above $c_{\mathrm{L}}^{\infty}$ at low $X$, which is unphysical and not predicted by the exact "Schlögl" model. This finalizes our brief discussion of Example II. 

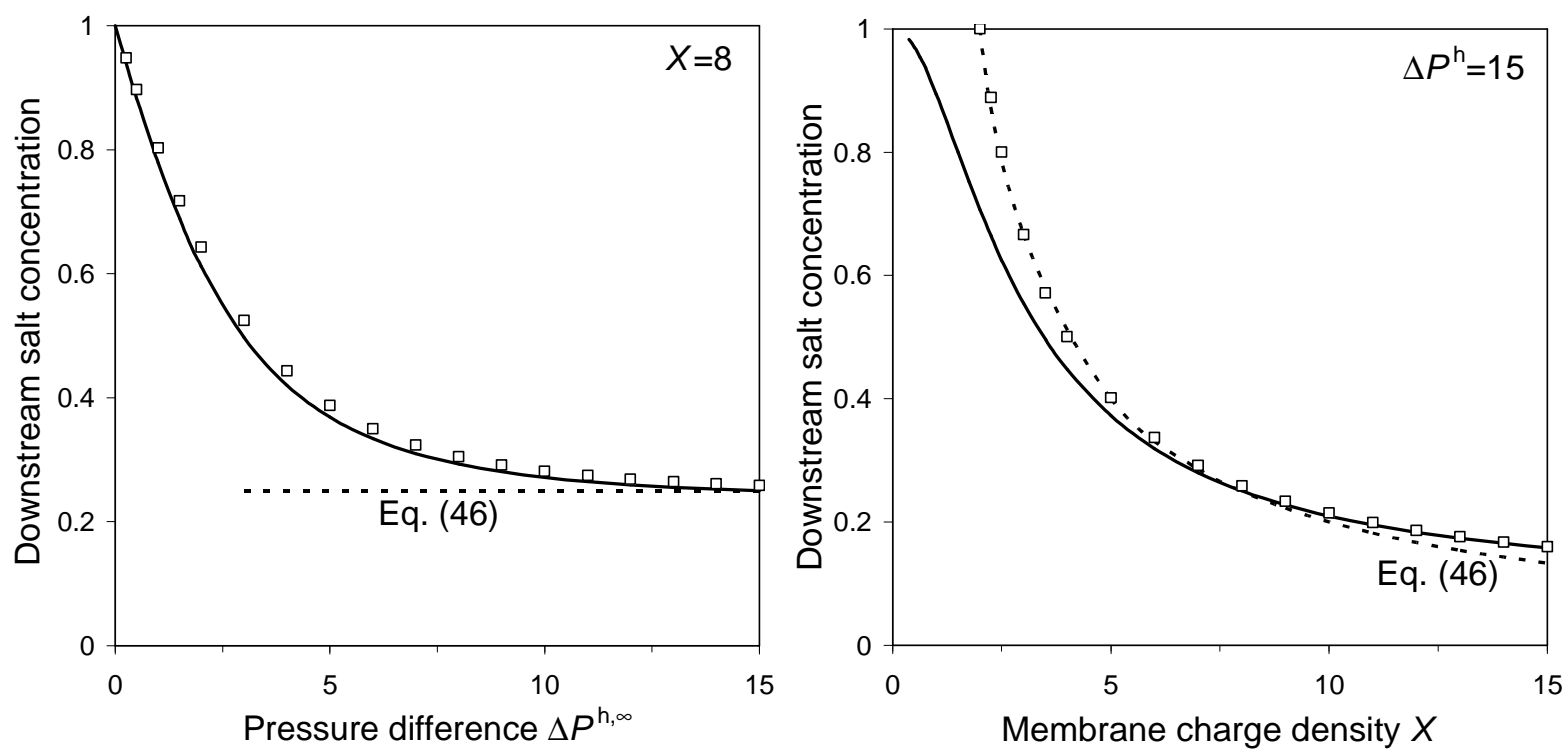

Fig. 2. Salt retention using an ion-exchange membrane in a batch-cell experiment. Solid lines: full model predictions given by Eqs. (30)-(40); squares: analytical model given by Eqs. (42) and (44); dashed line: analytical solution given by Eq. (46). (a). Downstream salt concentration $c_{\mathrm{R}}^{\infty}$ as function of applied pressure difference $\Delta P^{\mathrm{h}, \infty}(X=8)$ and (b). as function of membrane charge $X\left(\Delta P^{\mathrm{h}, \infty}=15\right)$. Parameter values for $X, \Delta P^{\mathrm{h}, \infty}$ and the downstream salt concentration, $C_{\mathrm{R}}^{\infty}$, must be multiplied by the value of the upstream concentration, $C_{\mathrm{L}}^{\infty}$, to become dimensional $\left(\Delta P^{\mathrm{h}, \infty}\right.$ must again be multiplied by $R T$ to arrive at dimension $\mathrm{J} / \mathrm{m}^{3}=\mathrm{Pa} ; f_{\mathrm{m}} \cdot D / c_{\mathrm{L}}^{\infty}=0.1$ ).

In conclusion, to simultaneously describe the transport of colloidal particles and the supporting fluid, including both the forces that act on the particles such as electrostatic field effects, and those that act on the fluid such as pressure, it is possible to implement a modified two-fluid equation - an approach which is very generally applicable to all kinds of processes in electrokinetics and membrane science. Though we have presented two example calculations for one-dimensional steady-state diffusion through a planar homogeneous membrane, the theory is equally well applicable to more complicated, e.g. dynamical, problems both in free solution and in porous media, taking also into account effects of solvation energy and particle volume.

\section{references}

1. R. Schlögl, Stofftransport durch Membranen, Band 9 of "Fortschritte der Physikalischen Chemie", Darmstadt (1964). pp. 57-77.

2. R. Schlögl, Z. Phys. Chemie NF 373 (1955).

3. E.H. Bresler and R.P. Wendt, Science 163, 944 (1969).

4. J.C. Fair and J.F. Osterle, J. Chem. Phys. 543307 (1971).

5. J.F. Osterle and M.J. Pechersky, J. Phys. Chem. 75, 3015 (1971).

6. E.N. Lightfoot, Transport phenomena and living systems, Wiley (1974).

7. A.A. Sonin, pp. 255-265 in Charged Gels and Membranes I, E. Sélégny (ed.) (1976).

8. E.H. Bresler and L.J. Groome, Am. J. Physiol. 241, F469 (1981).

9. L.F. Del Castillo and E.A. Mason, J. Membrane Sci. 28229 (1986).

10. E.A. Mason and H.K. Lonsdale, J. Membrane Sci. 511 (1990). 
11. P.J.A.M. Kerkhof, Chem. Eng. J. 64319 (1996).

12. D.C. Guell and H. Brenner, Ind. Eng. Chem. Res. 353004 (1996).

13. M.Z. Bazant, K. Thornton, and A. Ajdari, Phys. Rev E 70021506 (2004).

14. W. Sparreboom, A. van den Berg, and J.C.T. Eijkel, Nature Nanotechnology 4, 713 (2009).

15. M.Z. Bazant, M.S. Kilic, B.D. Storey, and A. Ajdari, Adv. Colloid Interface Sci. 15248 (2009).

16. P.M. Biesheuvel and M.Z. Bazant, Phys. Rev. E 81031502 (2010).

17. P.M. Biesheuvel and A. van der Wal, J. Membrane Sci. 346256 (2010).

18. R. Zhao, P.M. Biesheuvel, H. Miedema, H. Bruning, and A. van der Wal, J. Phys. Chem. Lett. 1, 205 (2010).

19. J.W. Post, H.V.M. Hamelers, and C.J.N. Buisman, Environm. Sci Techn. 42, 5785 (2008).

20. D. Brogioli, Phys. Rev. Lett. 103058501 (2009).

21. B.B. Sales, M. Saakes, J.W. Post, C.J.N. Buisman, P.M. Biesheuvel, and H.V.M. Hamelers, Env. Sci. \& Techn. 445661 (2010).

22. D. Brogioli, R. Zhao, and P.M. biesheuvel, Energy \& Environm. Sci. submitted (2010).

23. S.L. Ong, W. Zhou, L. Song and W.J. Ng, Env. Eng. Sci. 19429 (2002).

24. W.B.S. de Lint and N.E. Benes, J. Membrane Sci. 243365 (2004).

25. H. Strathmann, lon-exchange membrane processes, Elsevier (2004).

26. W. Zhou and L. Song, Env. Sci. Techn. 393382 (2005).

27. T.Y. Cath, A.E. Childress, and M. Elimelech, J. Membrane Sci. 28170 (2006).

28. K. Kontturi, L. Murtomäki, and J.A. Manzanares, Ionic Transport Processes, Oxford University Press (2008).

29. M.A. Shannon, P.W. Bohn, M. Elimelech, J.G. Georgiadis, B.J. Mariñas, and A.M. Mayes, Nature 452, 301 (2008).

30. R.J. Gross and J.F. Osterle, J. Chem. Phys. 49228 (1968).

31. P.M. Biesheuvel and J. Lyklema, J. Phys. Condens. Matter 176337 (2005).

32. P.M. Biesheuvel and M. van Soestbergen, J. Colloid Interface Sci. 316490 (2007).

33. P. Meares, pp. 123-146 in Charged Gels and Membranes I, E. Sélégny (ed.) (1976).

34. J.A.M. Kuipers, K.J. van Duin, F.P.H. van Beckum, and W.P.M. van Swaaij, Chem. Eng. Sci. 47, 1913 (1992).

35. B.P.B. Hoomans, J.A.M. Kuipers, W.J. Briels and and W.P.M. van Swaaij, Chem. Eng. Sci. 51, 99 (1996).

36. E. Delnoij, F.A. Lammers, J.A.M. Kuipers and W.P.M. van Swaaij, Chem. Eng. Sci. 521429 (1997).

37. R. Beetstra, M.A. van der Hoef and J.A.M. Kuipers, Chem. Eng. Sci. 62246 (2007).

38. J. Newman and K.E. Thomas-Alyea, Electrochemical Systems, $3^{\text {rd }}$ Ed., Wiley (2004).

39. P.M. Biesheuvel, H. Verweij, and V. Breedveld, AIChE J. 471969 (2001).

40. X. Yin and S. Sundaresan, AlChE J. 55, 1352 (2009).

41. H.C. Brinkman, Appl. Sci. Res. Al 27 (1947).

42. M.A. Cohen Stuart, F.H. Waajen, S.S. Dukhin, Colloid Polym. Sci. 262, 423 (1984).

43. J.F.L. Duval, R. Zimmermann, A.L. Cordeiro, N. Rein, and C Werner, Langmuir 25, 10691 (2009). 
44. J.H. Masliyah, Chem. Eng. Sci. 341166 (1979).

45. A. Mauro, Science 126252 (1957).

46. P.M. Ray, Plant Physiology 35, 783 (1960).

47. R.F. Probstein, Physicochemical Hydrodynamics, Butterworths, Boston (1989). pp. 193, 229.

48. C.L. Rice and R. Whitehead, J. Phys. Chem. 694017 (1965).

49. A. Szymczyk, H. Zhu, B. Balannec, J. Phys. Chem B. 11410143 (2010).

50. W.M. Deen, AIChE J. 33, 1409 (1987).

51. Y. Lanteri, P. Fievet, A. Szymczyk, J. Colloid Interface Sci. 331, 148 (2009).

52. M.A. Rutgers, J.H. Dunsmuir, J.-Z. Xue, W.B. Russel and P.M. Chaikin, Phys. Rev. B 535043 (1996).

53. O. Sten-Knudsen, Ch. 2 "Passive transport processes" in Membrane Transport in Biology, Vol. 1

D.C. Tosteson (ed.) Springer (1978).

54. G. Hertz, Z. Phys. 23433 (1922).

55. V. Sasidhar and E. Ruckenstein, J. Colloid Interface Sci. 82439 (1981).

56. N. Lakshminarayanaiah, Equations of membrane biophysics, Academic Press (1984).

57. S.G. Schultz, Basic principles of membrane transport, Cambridge University Press (1980).

58. A.J. Staverman, Trans. Faraday Soc. 48, 176 (1952).

59. O. Kedem and A. Katchalsky, Biochim. Biophys. Acta 27, 229 (1958).

60. A. Kargol, J. Membrane Sci. 19161 (2001).

61. K.S. Spiegler and O. Kedem, Desalination 1311 (1966).

62. J. Wei, Ind. Eng. Chem. 5855 (1966).

63. E.H. Bresler and R.P. Wendt, Science 1661438 (1969).

64. E.H. Bresler and R.P. Wendt, J. Phys. Chem. 73264 (1969).

65. E.H. Bresler, Hoppe-Seyler's Zeitschrift für Physikalische Chemie 35312 (1972).

66. E.H. Bresler, E.A. Mason, and R.P. Wendt, Biophys. Chem. 4229 (1976).

67. L. Axel, Bull. Math. Biol. 38671 (1976).

68. B.R. Locke, M. Acton, and S.J. Gibbs, Langmuir 17, 6771 (2001).

69. X. Lefebvre and J. Palmeri, J. Phys. Chem. B 109, 5525 (2005).

70. D.E. Goldman, J. Gen. Physiol. 2737 (1943).

71. T. Teorell, Prog. Biophys. Biophys. Chem. 3305 (1953).

72. T. Teorell, pp. 57-69 in Charged Gels and Membranes I, E. Sélégny (ed.) (1976).

73. F.G. Helfferich, Ion Exchange, Dover (1962), Ch. 8.

74. N. Lakshminarayanaiah, Transport phenomena in membranes, Academic Press (1969), p. 282.

75. J.G. Wijmans and R.W. Baker, J. Membrane Sci. 1071 (1995).

76. W.B.S. de Lint, P.M. Biesheuvel, and H. Verweij, J. Colloid Interface Sci. 251131 (2002).

77. W.R. Bowen and H. Mukhtar, J. Membrane Sci. 112263 (1996).

78. P. Baticle et al., J. Membrane Sci. 135, 1 (1997).

79. M.S. Hall, D.R. Lloyd, and V.M. Starov, J. Membrane Sci. 128, 39 (1997). 